\title{
WUSCHEL is a primary target for transcriptional regulation by SPLAYED in dynamic control of stem cell fate in Arabidopsis
}

\author{
Chang Seob Kwon, Changbin Chen, ${ }^{1}$ and Doris Wagner ${ }^{2}$ \\ Department of Biology, University of Pennsylvania, Philadelphia, Pennsylvania 19104, USA
}

\begin{abstract}
SNF2 chromatin-remodeling ATPases play an important role in ensuring proper development in higher eukaryotes by controlling accessibility of cis-regulatory DNA regions to transcription factors and to the transcriptional machinery. However, the biological targets controlled by these ATPases are largely unknown. Using genetic and molecular analyses we have identified WUSCHEL (WUS) as a biologically important target of the SNF2-class ATPase SPLAYED (SYD) in the shoot apical meristem of Arabidopsis. We present evidence that SYD is recruited to the WUS promoter and that it is involved in regulation of the stem cell pool maintenance via direct transcriptional control of this master regulator.
\end{abstract}

[Keywords: Chromatin-remodeling ATPase; shoot apical meristem; SWI/SNF; stem cell fate; epigenetic regulation; WUSCHEL]

Supplemental material is available at http://www.genesdev.org.

Received October 27, 2004; revised version accepted February 24, 2005.

Developmentally regulated gene expression is known to be controlled by evolutionarily conserved SWI/SNF ATPase complexes. Loss-of-function mutations in the central SNF2 ATPase subunits lead to a variety of developmental abnormalities (Tsukiyama 2002). Evidence is accumulating that SNF2 ATPases play important roles in cell division, differentiation, and embryo patterning in higher eukaryotes (Kennison and Tamkun 1988; Reyes et al. 1998; Bultman et al. 2000; Sawa et al. 2000; Wagner and Meyerowitz 2002; Gregg et al. 2003; Farrona et al. 2004).

Transcriptional regulation of many genes is dependent on SNF2-containing chromatin-remodeling complexes (Peterson and Workman 2000; Martens and Winston 2003), which control the access of trans-acting transcriptional regulators or of components of the general transcriptional machinery to the packaged eukaryotic genome (Emerson 2002; Narlikar et al. 2002). These complexes are recruited to cis-regulatory DNA regions by sequence-specific transcriptional activators/repressors or by specific histone modifications present at these sites (Peterson and Workman 2000; Martens and Winston

${ }^{1}$ Present address: Department of Biology, The Pennsylvania State University, University Park, PA 16802, USA.

${ }^{2}$ Corresponding author.

E-MAIL wagnerdo@sas.upenn.edu; FAX (215) 898-8780.

Article and publication are at http://www.genesdev.org/cgi/doi/10.1101/ gad.1276305.
2003). This recruitment results in ATP-dependent alteration of the nucleosome position on the DNA and/or weakening of the DNA-histone interaction (Becker and Horz 2002; Lusser and Kadonaga 2003).

A given developmental defect observed in loss-of-function mutants for individual SNF2 chromatin-remodeling ATPases could thus be caused by (1) the critical perturbation of the expression of a master regulator gene or (2) the summation of effects on expression of multiple target genes involved in the developmental process under investigation. Available experimental evidence aimed at identifying molecular targets of a chromatin-remodeling ATPase in other organisms did not distinguish between these possibilities (Holstege et al. 1998; Sudarsanam et al. 2000; Armstrong et al. 2002; Damelin et al. 2002; Ng et al. 2002). For instance, whole-genome analyses performed in yeast show that expression of $6 \%$ of all genes is dependent on the SWI2/SNF2 ATPase (Holstege et al. 1998; Sudarsanam et al. 2000), suggesting that expression of a substantial number of genes can be affected by the absence of a chromatin-remodeling ATPase. However, only a subset of these genes are expected to be direct targets. Moreover, it is not certain if all direct target genes are biologically important in the developmental process under investigation.

The Arabidopsis proteome contains 42 ATPases that have the seven diagnostic SWI2/SNF2 motifs (http:// www.chromdb.org). Phylogenetic analyses indicate that 
the Arabidopsis ATPases include representatives of all the conserved chromatin-remodeling subfamilies: SNF2, ISWI, CHD, DDM1, and SRCAP /Verbsky and Richards 2001; http://www.chromdb.org). Chromatin remodeling has been demonstrated for the Arabidopsis ATPase DDM1, suggesting that the Arabidopsis ATPases are functionally conserved with their yeast and/or mammalian counterparts (Brzeski and Jerzmanowski 2003). Four Arabidopsis genes are classified as members of the SNF2 subfamily of transcriptional coregulators (Verbsky and Richards 2001). In contrast, metazoans usually have one or two SNF2 genes, suggesting that the Arabidopsis ATPases may have more specific roles than their metazoan counterparts. In support of this idea, mutations in the single SNF2 ATPase in Drosophila cause lethality (Kennison and Tamkun 1988), while mutants of the Arabidopsis SNF2 ATPases SYD (Wagner and Meyerowitz 2002) and AtBRM (Farrona et al. 2004) are viable.

syd mutants have a variety of developmental defects, including short stature, slow growth, leaf polarity defects, ovule growth arrest, and loss of maintenance of the shoot apical meristem (SAM) (Wagner and Meyerowitz 2002; Eshed et al. 2004). This phenotypic pleiotropy is consistent with the idea that SNF2 chromatin-remodeling ATPases regulate transcription of genes involved in many developmental processes (Peterson and Workman 2000; Emerson 2002; Martens and Winston 2003). We were particularly interested in studying the basis for the shoot apical meristem defect in syd because a phenotypic defect is observed in the syd single mutant and because the signaling pathway that regulates SAM maintenance in plants is genetically well defined. This provides an opportunity to identify the specific targets of SYD regulation in SAM maintenance and to investigate whether the developmental defect in syd is the result of a defect in transcriptional regulation of a critical master gene such as SHOOT MERISTEMLESS (STM) and WUSCHEL (WUS), or of transcriptional misregulation of multiple SAM regulators.

Here we provide genetic and molecular evidence that SYD, a SNF2 class ATPase, plays a role in regulation of the stem cell pool in the SAM primarily via direct transcriptional control of the master SAM regulator WUS.

\section{Results}

\section{SAM defects observed in the syd-2-null mutant plants}

Adult syd plants undergo premature termination of the

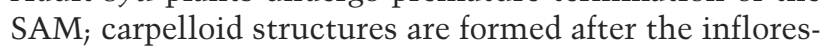
cence SAM has given rise to two to 20 flowers (Fig. 1C; Wagner and Meyerowitz 2002). In contrast, the wild type maintains the SAM until it senesces. In order to investigate when the SAM defect in the syd plants is first detectable, we compared SAM development in the syd2-null mutant with that in the Ler wild type at various developmental stages using scanning electron microscopy (SEM) (Fig. 1A). During the vegetative stage (at days 5 and 12), there were no discernable differences in SAM morphology between Ler and syd-2 plants. However, a
A

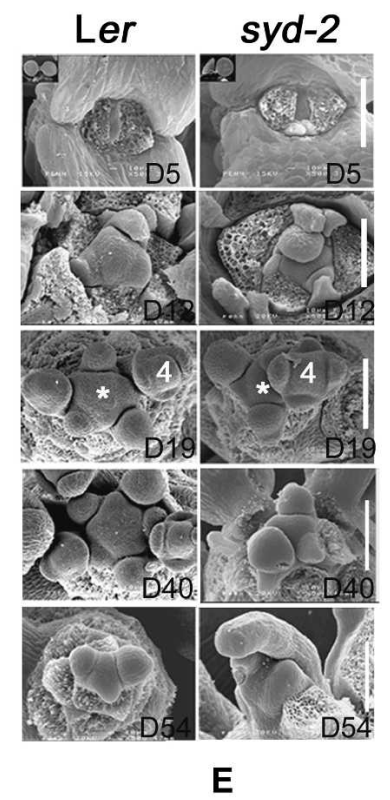

B
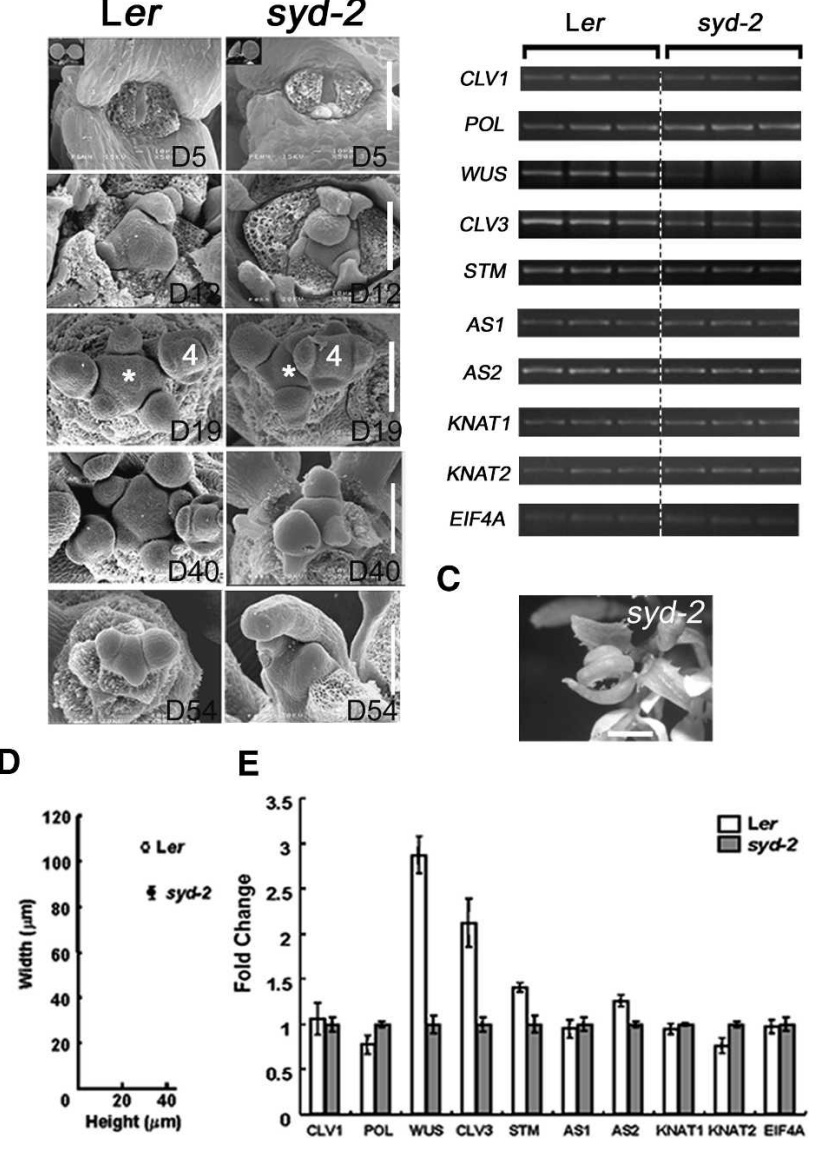

Figure 1. Morphological and molecular differences in the shoot apical meristem (SAM) between Ler and syd-2 plants. (A) Scanning electron microscope (SEM) images of the SAM in the wild-type Ler and syd-2 at different developmental stages. Days after germination are indicated. The SAM at day 19 is marked by white asterisks and stage 4 flowers are indicated (4). Bars, 100 $\mu \mathrm{m}$. (B) Semiquantitative RT-PCR analysis of known meristem regulators. Three independent RNA isolations each of wild-type (Ler) and syd-2 mutant seedlings were compared. RNA was isolated from the entire above-ground plant tissues $18 \mathrm{~d}$ after germination. (C) Photographs of syd-2 plants after 2 mo of growth show carpelloid structures produced after SAM termination while Ler plants retain their SAM until they senesce (data not shown). Bar, $1 \mathrm{~mm}$. (D) The width and height of the SAM in Ler and syd-2 plants at day 21 after germination were measured in histological sections. More than seven SAMs were used for measurements, and the standard error of the mean is shown. $(E)$ Quantification of message levels in $B$. The mean intensity of three independent RT-PCR products in syd-2 was set to 1 . The standard error of mean is shown. The genes abbreviated are CLAVATA (CLV), POLTERGEIST (POL), WUSCHEL (WUS), SHOOT MERISTEMLESS (STM), ASYMMETRIC LEAVES (AS), KNOTTED-like from ARABIDOPSIS THALIANA (KNAT), and Eukaryotic Translation Initiation Factor 4A-1 (EIF4A).

reduction in SAM size was evident in syd-2 during early reproductive development (day 19), and became even more pronounced at later stages of inflorescence development (day 40). The number of visible flower primordia at stage 4 or younger was reduced in syd-2 compared 
with Ler at day 19, suggesting a possible defect in SAM activity (Fig. 1A). Consistent with this, the width of the SAM was reduced in syd-2 plants at an early reproductive stage (day 21) (Fig. 1D). At day 54, the SAM in syd-2 plants was consumed, while Ler plants retained their SAM. Defects in the SAM are thus mainly apparent during the reproductive stage in syd-2-null mutants.

SAM maintenance is regulated primarily through two independent pathways by the homeodomain transcription factors WUS and STM /Clark 2001; Baurle and Laux 2003; Carles and Fletcher 2003). In one pathway, the size of the stem cell pool in the SAM is dynamically regulated by a negative feedback loop involving WUS and CLAVATA (CLV) (Brand et al. 2000; Schoof et al. 2000). WUS confers stem cell fate on the overlying cell layers in an unknown non-cell-autonomous manner (Laux et al. 1996; Mayer et al. 1998). The stem cells thus specified express the CLV3 protein ligand resulting in negative regulation of WUS through the CLV1/CLV2 leucine-rich repeat receptor kinase complex (Fletcher et al. 1999; Jeong et al. 1999; Trotochaud et al. 1999; Rojo et al. 2002; Lenhard and Laux 2003). The KNOX-class homeodomain transcription factor STM regulates a parallel pathway controlling the initiation and maintenance of the SAM (Long et al. 1996). STM maintains the SAM by preventing premature differentiation of meristem cells (Barton and Poethig 1993; Endrizzi et al. 1996). It does so by restricting the expression of ASYMMETRIC LEAVES1 (AS1) and ASYMMETRIC LEAVES2 (AS2) genes to organ primordia, where these genes are required for differentiation and for proper leaf development (Byrne et al. 2000; Tsiantis and Hay 2003). AS1 and AS2 negatively regulate the expression of KNOX genes, such as KNAT1 and KNAT2, which can compensate for the loss of STM in the absence of AS1 and AS2 (Byrne et al. 2000; Tsiantis and Hay 2003).

Since SYD, as a SNF2-class ATPase, is predicted to play a role in transcriptional regulation (Narlikar et al. 2002; Martens and Winston 2003), we compared the expression levels of known meristem regulators in 18-d-old wild-type (Ler) and syd-2 plants (Fig. 1B,E). At the stage during reproductive development when phenotypic defects in the SAM were first observed (Fig. 1A,D), the expression of most meristem regulators was not significantly altered (Fig. 1B,E). However, we detected a considerable reduction in the message levels of WUS and CLV3 in syd-2 relative to wild type. Consistent with the absence of a morphological difference between wild-type and mutant vegetative meristems, WUS and CLV3 expression was not reduced during the vegetative stage in syd mutants (data not shown; see Fig. 5A, below). Thus, the SAM defect in syd-2 during the reproductive stage is correlated with a reduction of WUS and of CLV3.

Genetic interaction between SYD and meristem-regulating transcription factors

To further test whether SYD regulates meristem maintenance via the WUS/CLV3 pathway rather than the STM pathway, we constructed the double mutants wus-1 syd-2, stm-2 syd-2, and stm-1 syd-2. Double mutants between wus-1 and weak stm alleles are known to result in strong meristem defects (Endrizzi et al. 1996) presumably because of the cooperative action of WUS and STM on SAM maintenance (Gallois et al. 2002; Lenhard et al. 2002).

If SYD acts in the STM pathway, we expect a similar phenotype in wus-1 syd-2 to that observed in the double mutant between wus-1 and a weak stm allele. In wus-1 plants, two young primary leaves were visible at day 14 (Fig. 2A,Q), and many irregular-shaped leaves (Fig. 2C) were formed from an abnormally flat meristem at later stages (Fig. 2D) as previously reported (Laux et al. 1996; Mayer et al. 1998). In contrast, no primary leaves were visible in wus-1 syd-2 plants at day 14 (Fig. 2E,R), suggesting a possible enhancement of wus-1 by syd-2. However, cotyledon petiole fusion was absent in wus-1 syd-2 in contrast to the strong petiole fusions observed in double mutants between wus-1 and a weak stm allele (Fig. 2R,S; Endrizzi et al. 1996), indicating that the phenotypic difference between wus-1 and wus-1 syd-2 is not derived from reduced STM function. Furthermore, closer observation by SEM analyses revealed the presence of a small, undifferentiated cell population (Fig. 2F) between the cotyledon petioles at this stage (day 12), suggesting presence of an active meristem in the double mutant. Consistent with this, small and partially radialized leaves developed in half of wus-1 syd-2 plants by day 28 (Fig. 2G,H; data not shown). This defect is consistent with a role of SYD in leaf polarity regulation (Eshed et al.

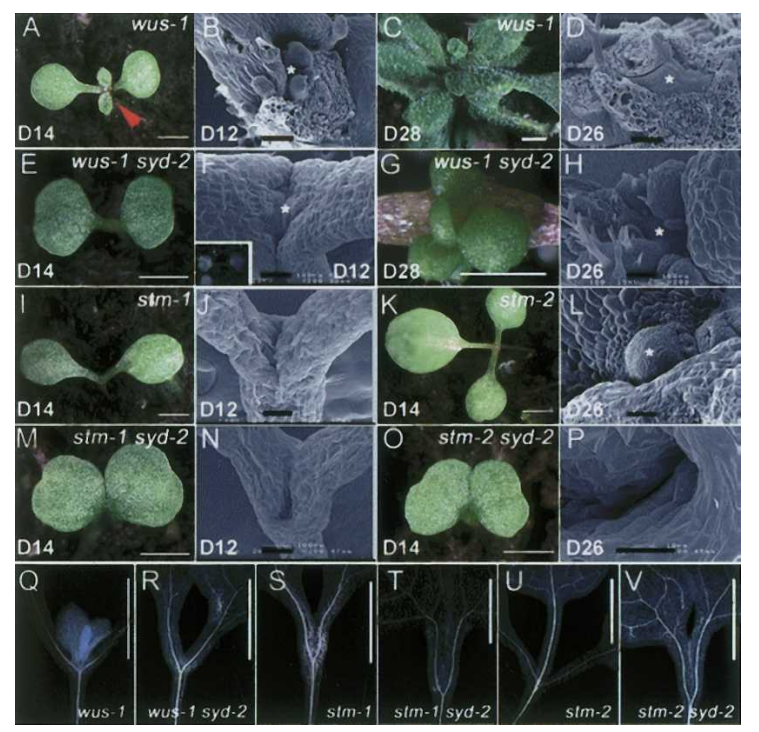

Figure 2. Genetic interactions between SYD and two homeodomain meristem regulators. Pictures of green plants and cleared seedlings were taken $14 \mathrm{~d}$ after germination except for $C$ and $G$ (28 d after germination). SEM images of the SAM were from 12-d-old plants $(B, F, J, N)$ or 26-d-old plants $(D, H, L, P)$. Shoot meristems are indicated with white asterisks. The two primary leaves produced in wus-1 (D14) are indicated by an arrowhead. Bars: in the green plant pictures, $2.5 \mathrm{~mm} ; G, 1 \mathrm{~mm}$; in the SEM images, $100 \mu \mathrm{m}$. Genotypes of green plants are indicated in the corresponding SEM images to the right. 
2004) and may contribute to the delayed leaf emergence and/or the reduced leaf size in wus-1 syd-2. Presence of residual meristem activity in wus-1 syd-2 as well as absence of cotyledon fusion in this double mutant together support the idea that SYD is not required for the STM SAM-maintenance pathway.

If SYD acts primarily in the WUS pathway, we expect a significant enhancement of a weak stm mutant by syd-2 (Endrizzi et al. 1996). Strong stm alleles exhibit near complete loss of SAM function and thus may only be marginally enhanced. In the weak stm-2 mutant a shoot meristem is initiated but arrested after making a few leaves (Fig. 2K), followed by repetitive initiation of defective shoot meristems (Fig. 2L), eventually resulting in a bushy adult plant (Clark et al. 1996). In contrast, stm-2 syd-2 never formed primary leaves (Fig. 2O,P; data not shown), indicating that syd-2 strongly enhances stm2. When we combined syd-2 with the stronger stm-1 mutant, no primary leaves were visible at day 14 in either stm-1, as previously reported (Barton and Poethig 1993), or in stm-1 syd-2 double mutants (Fig. 2I,J,M,N). While some stm-1 plants showed partial rescue of the meristem defect later in development (Clark et al. 1996), no phenotypic rescue could be detected in stm-1 syd-2 plants (data not shown), suggesting that stm-1 syd-2 plants have a more severe defect in meristem function than stm-1 plants. The combined genetic data are consistent with SYD acting primarily in the WUS pathway, as was suggested by the reduction of WUS and CLV3 in syd-2 compared with wild type (above).

\section{SYD is required for WUS transcription}

To verify the observed genetic interactions, we assayed gene expression levels in the single versus double mutants to evaluate the potential effects of syd on expression of WUS/CLV3 and STM. In stm-2 syd-2, a 12-fold reduction in WUS was observed compared with stm-2, while a lesser, but obvious, reduction occurred in CLV3 and in STM (Fig. 3A,B). The pronounced reduction of WUS (Fig. 3A,B) is likely the primary cause for the enhanced SAM defect in stm-2 syd-2. Because WUS is a positive regulator upstream of $C L V 3$, the reduction in $C L V 3$ could result from the reduced WUS activity. The observed reduction of $S T M$, on the other hand, is likely due to the reduced meristem size of stm-2 syd-2 (Fig. $2 \mathrm{O}, \mathrm{P})$. We conclude that SYD primarily regulates WUS expression.

Because of their near complete loss of SAM activity, stm-1 mutants have very low levels of WUS compared with wus-1 or stm-2 (Fig. 3A). While WUS expression was further reduced in stm-1 syd-2, this reduction was only twofold (Fig. 3A,B) possibly due to the already very low WUS expression in stm-1. The observed reduction in STM and CLV3 (Fig. 3A,B) is consistent with an enhancement of the SAM defect of $s t m-1$ by syd-2.

In wus-1 syd-2, we observed a very strong reduction in WUS message. Note that the wus-1 mutation (Mayer et al. 1998) causes a splicing defect (see Materials and

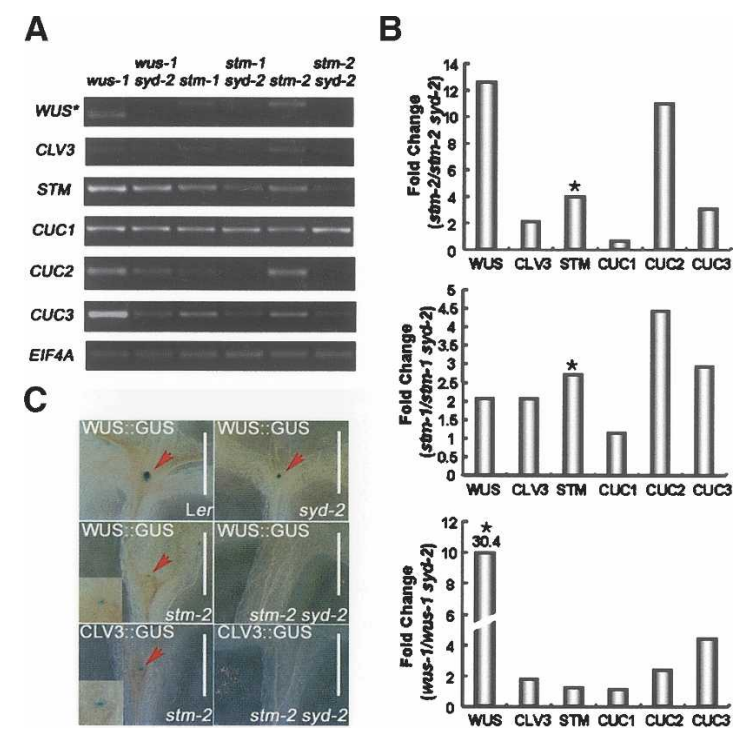

Figure. 3. Meristem-regulator expression in syd mutant meristems. (A) RT-PCR was performed using total RNA isolated from above-ground tissues of vegetative 12 -d-old seedlings. In stm-2 plants, a mature and expanded leaf was removed before RNA isolation because all other plants did not have mature leaves at this stage. The point mutation in wus- 1 causes the removal of the second exon resulting in a smaller transcript. $(B)$ Quantification of message levels. Intensities of the respective RT-PCR products from $A$ were normalized by the intensities of EIF4A. The fold change was obtained by dividing the signal strength in the single mutant by that of the double mutant. Mutant messages are indicated by black asterisks. (C) GUS analysis of WUS and CLV3 in 12-d-old wild-type and mutant plants. Strong GUS signals were detected in Ler and syd-2 plants after prolonged incubation $(16 \mathrm{~h})$ at $37^{\circ} \mathrm{C}$. The insets show a magnified view of the GUS-expressing cell population in stm-2 seedlings. The arrowhead indicates the GUS-expressing cell population. Bar, $1 \mathrm{~mm}$.

Methods), which results in a smaller transcript (Fig. 3A). This smaller transcript is expected to give rise to a truncated protein consisting of the $\mathrm{N}$-terminal 144 amino acids of WUS. The presumptive truncated protein includes the entire homeodomain. We also observed a slight reduction in CLV3 and STM message in wus-1 syd-2 compared with wus-1 (Fig. 3A,B). The marginal reduction in CLV3 and STM expression could suggest that SYD, in addition to regulating WUS, may play a minor role in other SAM-regulating pathways. Alternatively, if wus-1 is not a true null allele, the strong reduction of WUS-1 message by syd-2 could result in an additional reduction of SAM function (and hence CLV3 and STM expression) in this double mutant.

The reduction in WUS and CLV3 transcript levels in stm-2 syd-2 compared with stm-2 was confirmed by promoter activity analysis using GUS reporters. Both WUS: GUS and CLV3:GUS were detected in stm-2, whereas no GUS activity was detected in stm-2 syd-2 plants even after prolonged incubation with the substrate (Fig. 3C). We conclude that SYD regulates maintenance of the stem cell pool primarily via control of WUS expression. 
Kwon et al.

\section{SYD is required for cotyledon separation}

We noted a second phenotype in stm syd double mutants. These mutants exhibited strong cotyledon fusion (Fig. 2S-V). The fusion in stm-2 syd-2 exceeded that observed in stm-1, one of the strongest stm alleles (Fig. 2, cf. S and V; Barton and Poethig 1993). When we combined syd-2 with stm-1, enhanced cotyledon petiole fusion was observed compared with stm-1 (Fig. 2, cf. S and $\mathrm{T})$. Thus, in addition to SAM maintenance, SYD regulates cotyledon fusion. It is known that STM and the CUP-SHAPED COTYLEDON (CUC) genes can affect cotyledon separation (Aida et al. 1997, 1999; Takada et al. 2001; Vroemen et al. 2003). Because syd strongly enhances the cotyledon fusion defect in stm-1 and because SYD does not appear to act upstream of STM, SYD is not likely to control cotyledon separation via STM. We therefore monitored the expression of the CUC genes (CUC1, CUC2, and CUC3), which are implicated in cotyledon separation and organ boundary formation (Aida et al. 1997; Takada et al. 2001; Vroemen et al. 2003), in the double mutants. SYD regulation of these genes may be correlated with the enhanced cotyledon fusion we observed in stm-2 syd-2 and stm-1 syd-2 plants (Fig. 2T, V). Indeed, we observed a strong reduction in the message abundance of CUC2 and CUC3 but not of CUC1 in these mutants, suggesting a selective requirement for SYD in CUC expression (Fig. 3A,B). Absence of either CUC1 or CUC2 function in the background of stm mutants is known to enhance cotyledon fusion (Aida et al. 1999). Therefore, the reduction of transcript levels in CUC2 and CUC3 in stm-2 syd-2 and stm-1 syd-2 (Fig. 3A,B) is likely the main cause for the strong enhancement of cotyledon fusion in these mutants (Fig. $2 \mathrm{~T}, \mathrm{~V})$. The reduction of CUC2 and CUC3 messages is not likely to contribute to the SAM defect of syd mutants because CUC genes affect SAM function through STM (Takada et al. 2001), while SYD does not appear to regulate STM (see above).

Taken together our findings support the conclusion that SYD acts upstream of WUS in the regulation of stem cell fate. In addition, we showed that SYD plays a separate role in cotyledon separation via regulation of CUC2 and CUC3 but not CUC1 expression.

\section{clv1-4 partially rescues the SAM defect of syd-2}

The CLV1 receptor regulates WUS levels via a negative feedback loop. In clv1 mutant plants, increased WUS transcription results in increased accumulation of stem cells and a subsequent increase in the size of the SAM (Clark et al. 1993, 1997; Fletcher et al. 1999; Brand et al. 2000; Schoof et al. 2000). The specificity of the effect of CLV1 on WUS and the opposite phenotypes of clv1 and syd mutants allowed us to further investigate whether SYD acts on WUS via CLV and the negative feedback loop.

We constructed double mutants between syd-2 and clv1-4, a very strong dominant-negative allele (Dievart et al. 2003), to investigate the genetic interaction of these proteins in SAM function (Fig. 4). The overall plant morphology of clv1-4 syd-2 was similar to that of syd-2 rather than that of clv1-4, indicating that other roles of SYD (e.g., in leaf and flower patterning) were not affected by clv1-4 (Fig. 4A). However, we observed a striking difference in SAM morphology between clv1-4 and clv1-4 syd-2 plants during the vegetative stage. The SAM was enlarged in clv1-4 seedlings but not in clv1-4 syd-2 or wild-type seedlings (Figs. 4C, 1A). Later in development, overproliferation of the SAM was observed in clv1-4 and clv1-4 syd-2 (Fig. 4C, day 26). However, clv1-4 syd-2 meristems tended to fasciate rather than to expand radially

A

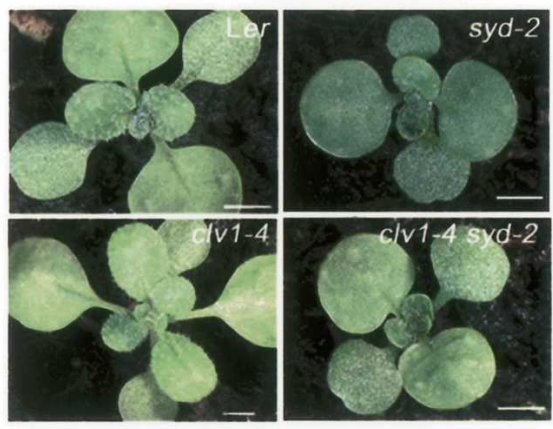

B
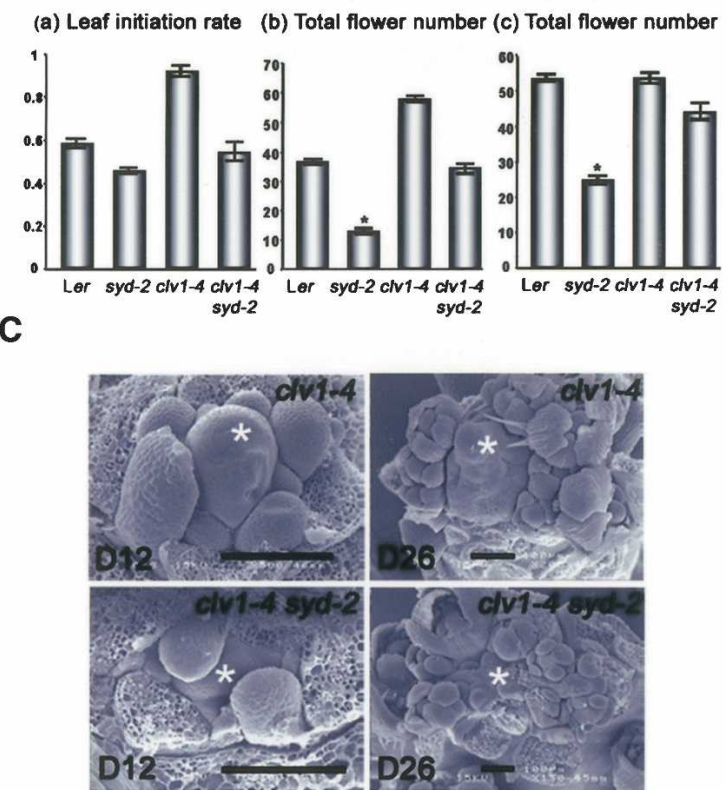

Figure 4. Phenotypic comparison between clv1-4 and clv1-4 syd-2. (A) Photographs of green plants taken $14 \mathrm{~d}$ after germination. $(B)$ Leaf initiation rate (graph $a$ ) and total flower number produced (graphs $b, c$ ) under long day (graphs $a, c)$ and continuous light (graph $b$ ) are shown. (Graph $a$ ) Leaf initiation rate is calculated as the number of new leaves produced per day between the fourth and the seventh true leaf. (Graphs $b, c$ ) Total flower number refers to flowers stage 12 and above. Black asterisks in $B$ indicate termination of the SAM. (C) SEM images of the SAMs in single versus double mutants. Developmental time points are indicated. White asterisks in $C$ indicate the SAM during vegetative and reproductive stages. Bars: $A, 2.5 \mathrm{~mm} ; C, 100 \mu \mathrm{m}$. 
like clv1-4 meristems. This linear expansion may be viewed as a less pronounced SAM overproliferation (Clark et al. 1993, 1996). A large number of flower primordia initiated from the linearly enlarged SAM in clv1-4 syd-2, suggesting at least partial rescue of the SAM defect of syd-2 by clv1-4 (cf. Figs. 1A and 4C).

We next measured leaf initiation rate and counted total flower number (Fig. 4B) in an attempt to investigate SAM activity in wild-type and mutant plants. Leaf initiation rate and total flower number were increased in clv1-4 compared with wild type in agreement with the increased SAM size in the mutant. The leaf initiation rate and total flower number of clv1-4 syd-2 was markedly lower than that of clv1-4 but higher than that of syd-2 (Fig. 4B), indicating that the SAM activity of clv1-4 syd-2 is intermediate between clv1-4 and syd-2. The intermediate phenotype in clv1-4 syd-2 indicates that SYD does not regulate WUS levels via inhibition of the CLV negative feedback loop.

It should also be noted that clv1-4 rescues the SAM termination of syd-2 plants during the reproductive phase (Fig. 4B, graphs b,c). Thus the reduced WUS activity in syd-2 may be an important cause for the SAM maintenance defect in the mutant.

\section{SYD is a limiting factor for elevated WUS transcription in clv1-4}

CLV1 is a negative regulator of WUS and of the stem cell pool, a collection of undifferentiated cells that express $C L V 3$. To ascertain whether the antagonistic interaction between clv1-4 and syd-2 results from opposite effects on WUS transcription, we investigated the message levels of WUS, CLV3, and STM in the mutants (Fig. 5A,B). In clv1-4 plants, the WUS message is strongly increased compared with that of the wild type, consistent with the inhibitory role of CLV on WUS transcription. However, this increase in WUS expression was sharply reduced in the absence of SYD (Fig. 5A), indicating that SYD is a limiting factor for elevated WUS transcription in clv1-4. The reduction of WUS message levels in clv1-4 syd-2 was more pronounced than that of all other genes tested (Fig. 5B), suggesting that SYD primarily affects WUS expression. Consistent with the role of WUS as an inducer of CLV3 (Fletcher et al. 1999; Brand et al. 2000, 2002; Schoof et al. 2000), CLV3 expression is reduced in clv1-4 syd-2 compared with clv1-4; however, this reduction is much less pronounced than that of WUS (Fig. 5A,B). We also compared WUS expression in clv1-4 versus clv1-4 syd-2 using the WUS:GUS transgene as a reporter. We observed a strong reporter signal in a broad domain within the enlarged SAM of clv1-4 seedlings (indicated by arrowheads in the light micrographs of a cleared seedling and of a histological section in Fig. 5C). In contrast, a weak WUS:GUS signal was detected in the center of the comparatively small SAM of clv1-4 syd-2 plants (indicated by an arrowhead in the light micrograph of a cleared seedling in Fig. 5C). Both the size of the WUSexpressing domain as well as staining intensity appear to be reduced in clv1-4 syd-2 compared with clv1-4 (Fig.

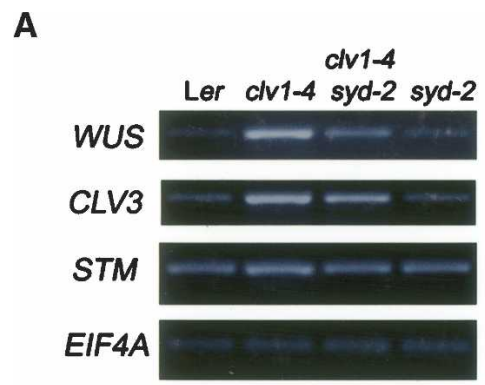

$\mathbf{B}$

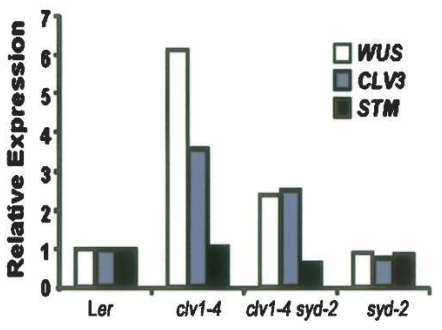

C

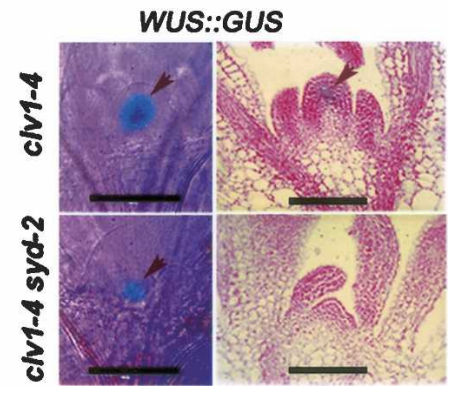

Figure 5. SYD is a limiting factor for WUS transcription in clv1-4. (A) RT-PCR was performed to investigate the message abundance of WUS, CLV3, and STM. (B) The band intensities of the respective messages from $A$ were normalized to the band intensities of EIF4A, and the normalized band intensities in Ler were set to one arbitrary unit. (C) GUS assays were performed in 8-d-old clv1-4 and clv1-4 syd-2 seedlings harboring a GUS transgene driven by the WUS promoter. Incubation with substrate was performed for $90 \mathrm{~min}$ at $37^{\circ} \mathrm{C}$. GUS signals are shown from cleared plant seedlings (left photographs) and sectioned plants (right photographs). Bars, $100 \mu \mathrm{m}$.

5C), suggesting that the pool of WUS-expressing cells as well as the level of WUS expression per cell is reduced in clv1-4 syd-2.

In summary, the reduction in SAM size and activity in clv1-4 syd-2 versus clv1-4 is correlated with strongly reduced levels of WUS transcript in the double mutant, consistent with the proposed role (above) for SYD in stem cell maintenance via regulation of WUS transcript levels.

\section{SYD is recruited to the promoter region of WUS}

Dependence of WUS message levels on SYD in a variety of SAM-mutant backgrounds and the molecular identity of SYD as a SNF2 ATPase suggest that SYD may act 
directly on WUS transcription as a chromatin-remodeling factor through interaction with sequence-specific transcriptional activators. This hypothesis can be tested by chromatin immunoprecipitation (ChIP) assays, which monitor in vivo binding of regulatory factors to nucleosomal DNA (Orlando 2000). This method relies on immunoprecipitation of a protein bound (and cross-linked) to DNA, followed by PCR amplification of the precipitated DNA to test for enrichment compared with control immunoprecipitations using preimmune serum and relative to nonprecipitated (input) genomic DNA. Indeed, significant enrichment of genomic DNA harboring a defined cis element has been observed for ATP-dependent chromatin-remodeling factors in yeast and mammalian cell cultures using this technique (Orlando 2000; Damelin et al. 2002; Ng et al. 2002; Kadam and Emerson 2003). However, WUS is expressed in $<10 \%$ of the cells of the SAM and $<1 \%$ of the cells in young seedlings (Fig. 1; Mayer et al. 1998). In order to test whether SYD can be specifically recruited to the genomic WUS region for transcriptional activation, we therefore chose to use inflorescence tips of clv1-4 because of the enlarged domain of WUS-expressing cells in this genotype and because WUS expression in this background is dependent on SYD (Figs. 4, 5).

For the ChIP we used a SYD-specific polyclonal antibody generated against the $\mathrm{N}$-terminal domain (upstream of the ATPase) of SYD (Fig. 6B; Y. Su and D. Wagner, unpubl.). This antibody detects a prominent band in nuclear extracts of wild-type inflorescences, but not in those of syd-2 mutants (Fig. 6B). We used a commercially available antibody against RNA polymerase II (RPO) to ensure equal loading of nuclear extracts from both wild-type and mutant inflorescences in this experiment (Fig. 6B).

Compared with the input and the preimmune serum control, the SYD antibody ChIP enriched the proximal WUS promoter region (site $\mathrm{P}$ ) but only weakly precipitated the transcribed region of WUS (site G) in agreement with the presumed role of SYD as a coactivator of transcription (Fig. 6A). Relative to the input, the genomic regions further upstream of the WUS coding region were less strongly enriched (Fig. 6A, site D), suggesting that SYD preferentially associates with WUS promoter-proximal elements. This is consistent with the preferential association of SWI2/SNF2 ATPase with promoters rather than coding regions in target genes $(\mathrm{Ng}$ et al. 2002). In contrast, the SYD antibody ChIP did not enrich STM promoter-proximal (Fig. 6B, site P) or promoter-distal regions (Fig. 6B, site D) compared with the coding region (Fig. 6B, site $\mathrm{G}$ ). These data suggest that SYD is specifically recruited to the WUS promoter.

As a control for protein occupancy at the sites tested and for ability to immunoprecipitate the two nucleosomal DNA templates, we next assayed for the presence of RNA polymerase II (RPO) at the WUS and the STM promoters. The proximal promoter regions (site $\mathrm{P}$ ) of WUS and STM as well as the transcribed regions (site G) were both similarly enriched in ChIP experiments with the RPO antibody (Fig. 6), consistent with the role of
A

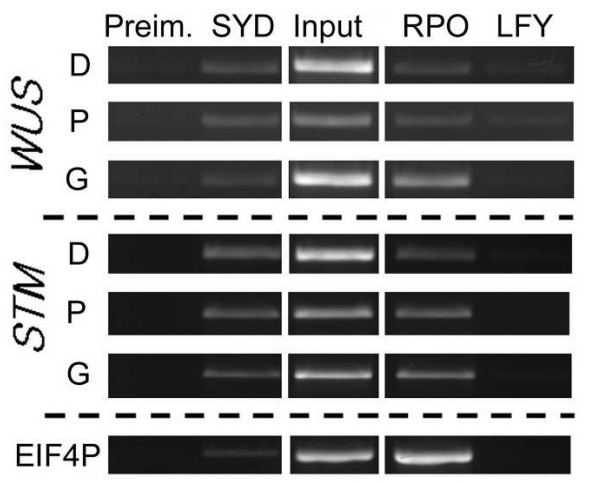

B
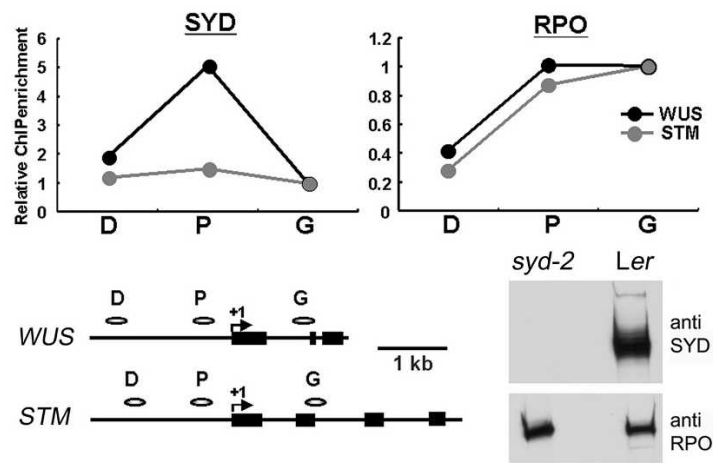

Figure 6. Recruitment of SYD to the WUS promoter. (A) Chromatin immunoprecipitation (ChIP) was performed with antibodies against SYD, RNA polymerase II (RPO), and LEAFY (LFY). Genomic regions amplified are indicated on the left. (D) Distal region; $(\mathrm{P})$ proximal promoter region; $(\mathrm{G})$ gene/coding regions. Precipitations were performed with preimmune serum (Preim.), SYD-specific antiserum (SYD), anti-RNA polymerase II antibodies (RPO), and anti-LEAFY antibodies (LFY). Genomic DNA $(0.1 \%)$ was also amplified with each primer set (Input). (B, top) Relative ChIP enrichment represents the normalized ratios of the precipitated genomic DNA compared with $0.1 \%$ nonprecipitated genomic DNA (Input) after first subtracting the value of nonspecific precipitation by preimmune serum. The value of ChIP enrichment in the coding region (G) was set to 1 for both WUS and STM. SYD-specific antiserum (SYD) (left panel) and anti-RNA polymerase II antibodies (RPO) (right panel) were used for precipitation. ( $B$, bottom left) Schematic diagram of the WUS and STM locus spanning promoter and coding regions. The WUS and STM open reading frame is shown by black boxes and the translation initiation site is indicated (arrow). The DNA regions amplified by PCR are shown above the genomic structure. ( $B$, bottom right) Western analysis of nuclear extracts from wild-type and syd-2 inflorescences probed with SYD (top panel) and RPO antibodies (bottom panel). SYD protein is detected strongly in wild type but absent in the syd-2-null mutant.

RNA polymerase in transcriptional initiation and elongation. This supports the interpretation that SYD preferentially binds to the proximal promoter region of WUS. To further examine the specificity of the SYD recruitment, we assayed for SYD binding to the promoter region of the eukaryotic translation initiation factor $4 \mathrm{~A}$ (EIF4A). EIF4A is ubiquitously expressed, and its mes- 
sage abundance is not dependent on SYD (Figs. 1, 3, 5). Hence EIF4A is not expected to be bound by SYD (Fig. 5). Relative to the input, we detected very weak association of SYD with the proximal promoter region of EIF4A (Fig. 6, EIF4P). Consistent with the ubiquitous expression of EIF4A, we detected very strong binding of RPO to this promoter (Fig. 6, EIF4P). Thus, SYD does not nonspecifically associate with promoters of transcriptionally active genes.

We noted considerable basal enrichment throughout the genomic regions tested in immunoprecipitation using SYD and RPO antibodies compared with those using preimmune serum (Fig. 6A), which is possibly due to an inherent nonspecific affinity of SYD and RNA polymerase to nucleosomal DNA. The RSC SWI2/SNF2 complex in yeast, for example, interacts nonspecifically with DNA and nucleosomes in vitro (Lorch et al. 1999; Sengupta et al. 2001). Consistent with this interpretation, the amount of DNA immunoprecipitated with an antibody against the LEAFY transcription factor (William et al. 2004) was comparable to that of the preimmune ChIP at most genomic sites (Fig. 6A).

We conclude that SYD is specifically recruited to the proximal promoter region of WUS in the SAM of clv1-4 inflorescences. This indicates that the transcriptional regulation of WUS by SYD, which is important for control of stem cell fate, is direct.

\section{Discussion}

Many studies have focused on identifying molecular targets of a chromatin-remodeling ATPase (Holstege et al. 1998; Sudarsanam et al. 2000; Armstrong et al. 2002; Damelin et al. 2002; Ng et al. 2002). However, genetic analyses are required for identification of the biologically relevant target(s) and hence to understand the role of chromatin-remodeling ATPases in development. Using genetic and molecular analyses, we show here that WUS, a central regulator of stem cell fate, is a biologically relevant, direct target of SYD for SAM maintenance. This makes WUS the first direct target of a chromatin-remodeling ATPase in the plant kingdom. Furthermore, our results suggest that a specific developmental defect resulting from the loss of activity of a chromatin-remodeling ATPase can largely be attributed to perturbation of the expression of a single master regulator.

Here we investigated how SYD regulates SAM maintenance. We show that the meristem maintenance defect of the syd phenotype is not the result of expression perturbation of multiple target genes as a consequence of loss of SYD function. Instead, WUS, a central regulator of SAM maintenance, was found to be a functionally relevant target of SYD in the control of stem cell fate in the SAM. SYD acts in the WUS pathway because the SAM defect in wus was not substantially enhanced by syd, indicating that loss of SYD activity does not strongly affect meristem regulators in other meristemregulating pathways. In support of this, we also observed a strong reduction of WUS transcript in syd-2 single mutants during the reproductive phase and in several syd- 2-based double mutants. Furthermore, chromatin immunoprecipitation experiments indicate that SYD is specifically recruited to the proximal promoter region of the WUS locus, consistent with the genetic data and with the identity of SYD as a putative chromatin-remodeling factor. The antagonistic effects of CLV1 and SYD on SAM function indicate that SYD positively regulates the stem cell pool. In addition, the intermediate SAM phenotype of clv1-4 syd-2 compared with each single mutant suggests that SYD does not regulate WUS via inhibition of the CLV pathway.

We do not exclude the possibility that SYD may have additional, minor, roles on expression of other meristem regulators such as CLV3. In fact, the expression domain of SYD in the SAM encompasses the expression region of CLV3 and STM (Wagner and Meyerowitz 2002). The slight reduction of CLV3 message levels in wus-1 syd-2 compared with wus-1 suggests that SYD may regulate CLV3 expression directly or through an unknown WUSredundant stem-cell-promoting pathway. However, any involvement of SYD in transcriptional regulation of these other potential targets is unlikely to contribute substantially to the SAM maintenance defect resulting from loss of SYD activity because the single- and doublemutant phenotypes are explained by and correlate with a strong reduction in WUS expression.

Additional findings support a role of chromatin regulation in control of WUS expression. For instance, variegated and ectopic expression of WUS is observed in loss-of-function mutations of FAS1 or FAS2 and BRU1, which are implicated in chromatin assembly and epigenetic silencing, respectively (Kaya et al. 2001; Takeda et al. 2004). The altered WUS expression is correlated with overproliferation and fasciaton of the SAM. The data suggest that a restrictive epigenetic mark is present on the histone tails or on the DNA at the WUS locus in WUS nonexpressing cells of the SAM (but not in the organizing center), which is lost stochastically in these mutants, such that WUS transcription occurs in ectopic patches. However, the identity of this putative epigenetic mark and the reason for the requirement for FAS and/or BRU1 is currently not understood. In addition, the expression domain of WUS and $A G$ is abnormally expanded in floral meristems in a mutant of AtGCN5, a histone acetyltransferase related to yeast GCN5 (Bertrand et al. 2003). It is unclear whether AtGCN5 directly restricts the expression of WUS, especially given that histone acetyltransferases usually act as transcriptional coactivators (Peterson and Workman 2000; Narlikar et al. 2002). Thus, spatially restricted expression of WUS in the SAM is controlled via chromatin regulation. In contrast, SYD is required for proper WUS transcript levels in its normal expression domain, the organizing center. syd mutants do not exhibit fasciation or ectopic WUS expression. A single focus of WUS:GUS-expressing cells was observed in the SAM of clv1-4 syd-2 mutants. Both the domain of WUS-expressing cells and the WUS:GUS signal intensity per cell were reduced in the organizing center of clv1-4 syd-2 compared with that of clv1-4. Furthermore, the organization of the cell layers in the SAM 
is not perturbed in syd mutants. These observations suggest that SYD regulates WUS expression differently from FAS1 or FAS2 and BRU1.

The finding that SYD is required for up-regulation of WUS transcription is consistent with the idea that SNF2-class ATPases are mainly involved in inducible or increased transcription (Narlikar et al. 2002). Two general models could explain the functional requirement for SYD in transcriptional up-regulation of WUS (Supplementary Fig. S1). In one scenario the nucleosomal DNA in the promoter region of WUS is not accessible to transcriptional activators or to the general transcriptional machinery in the absence of chromatin remodeling. In this case, the fact that SAM function is only impaired late in syd-null mutants is likely due to the activity of another, partly redundant, chromatin-remodeling activity. In this model, the second ATPase would provide the local change in nucleosomal structure in the WUS promoter in the absence of SYD, albeit less efficiently, thus resulting in lower WUS transcript levels. This model is supported by the fact that four SNF2 ATPases are present in Arabidopsis (Verbsky and Richards 2001). Moreover, the closest homolog to SYD, AtBRM, has recently been reported to play a role in SAM maintenance (Farrona et al. 2004). Alternatively, it is possible that the chromatin architecture of the WUS locus is partially "open" such that a low level of WUS expression is independent of ATP-dependent chromatin-remodeling activity. In this model elevated WUS transcription requires a structural change in the nucleosomal DNA driven by the SYDcontaining chromatin-remodeling complex for enhanced binding of the same or of additional transcriptional activator(s). Both models are consistent with our observation that SYD is not required for WUS transcription under developmental conditions that do not require high levels of WUS message such as during the vegetative stage in the wild type. In addition, these models explain why syd causes SAM maintenance defects upon transition to reproductive development or under other conditions that may create or require increased levels of WUS transcript (in clv1 or wus/stm mutants, respectively).

In summary, we show here that SYD is a direct and specific positive upstream regulator of WUS expression in the organizing center and that this activity is required for proper stem cell maintenance in the Arabidopsis shoot apical meristem.

\section{Materials and methods}

\section{Plants}

stm-1, clv1-4, stm-2, wus-1, and syd-2 mutants have been described (Barton and Poethig 1993; Clark et al. 1993, 1996; Laux et al. 1996; Wagner and Meyerowitz 2002). Transgenic Arabidopsis seeds harboring WUS:GUS and CLV3:GUS were kindly provided by M. Lenhard and T. Laux (Institut für Biologie III, Universität Freiburg, Freiburg, Germany) (Groß-Hardt et al. 2002), and introgressed into the appropriate mutant background. Double-mutant plants were genotyped for syd-2, wus-1, and stm-2 using dCAPS (Neff et al. 1998) and maintained as double heterozygotes. Heart-shaped cotyledons served as a visible marker for syd-2 in young seedlings.
Arabidopsis seeds were sown on fertilized soil mix (Promix BX; Premier Horticulture), stratified in the dark at $4^{\circ} \mathrm{C}$ for $7 \mathrm{~d}$ and then grown at $22^{\circ} \mathrm{C}$ in long day $(16 \mathrm{~h} \mathrm{light})$ at $120 \mu \mathrm{mol} / \mathrm{m}^{2}$ sec of cool white light. Plants were photographed using an Olympus SZX12 dissecting microscope equipped with a Spot Insight camera (Diagnostic Inc.).

Reverse-transcriptase-mediated polymerase chain reaction ( $R T-P C R$ )

All above-ground plant tissues were used for RNA isolation. RNA isolation, reverse transcription, and PCR conditions were as described (William et al. 2004). PCR cycles varied from 26 to 36 depending on the message level. The PCR primers used are WUS, ACAAGCCATATCCCAGCTTCA and CCACCGTTG ATGTGATCTTCA; CLV1, GCTTGCTAAGTTCTTAGTTGA TGG and GCATCCGATGGCTGAGTTAT; CLV3, AATGGAT TCTAAAAGCTTTGTGCT and ACACCAAACGAAACAGAT TGC; $P O L$, GTACCACCGACCGTAGTGCT and GTGACGA TCATGGAGCCTTT; STM, CATAACGAAATCGTTGCAG GA and ATCGACTTCTTCCTCGGATGA; AS1, TGTGAT GGAAGTTGCTCTTGA and ACCACTTCCCTAACCGCT TT; AS2, TCCCATCTGTTTTGTTCCCTA and TCTGAAGC TGACGAAGCTGA; KNAT1, TCTCCCTCAAACAACCGA TAA and TGGGACTCTGACACAACATG; KNAT2, ACCAC CGGAGACAATCAAAG and TCCGCTGCTATGTCATCA TC; CUC1, CCACCTGGGTTTAGGTTTCA and AGAACCG GCAGAGGAGAACT; CUC2, TATCTTCCACCGGGTTTC AG and CCGGTGCTAGCTAAAGTGGT; CUC3, CTCGAA AACGACCATTCACA and CGTTGAGCTGAGAGACGACA; EIF4A, AAACTCAATGAAGTACTTGAGGGACA and TCT CAAAACCATAAGCATAAATACCC.

\section{Cloning and sequencing of PCR product}

An RT-PCR product harboring the wus-1 mutation was subcloned into pGEM-T Easy vector (Promega), and sequenced using an automated sequencer. The wus-1 mutation in the splicing acceptor site of the first intron resulted in the removal of the $\sim 90$-nt second exon by joining the donor site of the first intron to the acceptor site of the second intron (data not shown).

\section{$\beta$-glucuronidase (GUS) assays and clearing of plant seedling}

Arabidopsis seedlings were fixed overnight at room temperature in the solution of $90 \%$ ethanol and $10 \%$ acetic acid. After rehydration in $90 \%, 70 \%$, and $50 \%$ ethanol solution for $20 \mathrm{~min}$ each, seedlings were cleared with Hoyer's medium (Liu and Meinke 1998). Cleared seedlings were photographed against a dark background using a Leica MZFLIII stereo microscope equipped with a CoolSnap camera (Photometrics Inc.). GUS staining and histological sections were performed essentially as described (Sieburth and Meyerowitz 1997). Arabidopsis seedlings harboring homozygous GUS transgenes were fixed in $90 \%$ ice-cold acetone, incubated with substrate for the durations indicated in the figure legends, and cleared for photography. For histological sections of GUS-stained plants, we used tertiary butanol instead of xylene. GUS signals were photographed using an Olympus BX51 compound microscope.

\section{Scanning electron microscopy (SEM)}

Tissue fixation and scanning electron microscopy were previously described (Chen et al. 2000). Samples were examined with a Jeol 6400 analytical scanning electron microscopy. Digital images were edited using Photoshop 6.0 (Adobe). 


\section{Western analysis}

Nuclear extracts prepared from frozen plant tissues (Moehs et al. 1988) were dissolved in $1 \times$ Laemmli SDS sample buffer and run on $4 \%-12 \%$ gradient gel (Jule, Inc.). Membranes were probed with either anti-RNA polymerase II antibody generated against wheat germ Pol II (1:500; Covance) or SYD N-termspecific antiserum $(1: 10,000)$ (Y. Su and D. Wagner, unpubl.), followed by secondary probing with anti-mouse HRP $(1: 2,500)$ or anti-rabbit HRP $(1: 10,000)$, respectively. Secondary antibodyHRP conjugates and the detection kit (ECL plus) were from Amersham Biosciences.

\section{ChIP}

Buffers and procedures were essentially based on a manufacturer's protocol (Upstate). Plant tissue ( $300 \mathrm{mg})$ was cross-linked in $1 \%$ formaldehyde solution under vacuum for $15 \mathrm{~min}$. After incubation in $0.125 \mathrm{M}$ glycine for $5 \mathrm{~min}$, plant tissues were washed twice with $1 \times$ PBS, and subjected to preparation of nuclear extracts (Moehs et al. 1988). Nuclei were lysed in SDS Lysis Buffer (Upstate), diluted 10-fold with ChIP Dilution Buffer (Upstate), and sonicated to be a size range of $0.3-1.5 \mathrm{~kb}$. Antibodies were prebound to protein A magnetic beads (Dynal Biotech) by overnight incubation at $4^{\circ} \mathrm{C}$ in $1 \times$ PBS with $0.5 \%$ BSA. Antiserum against LFY was described previously (William et al. 2004). Immunoprecipitation was performed in ChIP Dilution Buffer containing $2 \mu \mathrm{g} / \mathrm{mL}$ salmon sperm DNA for $16 \mathrm{~h}$ at $4^{\circ} \mathrm{C}$ on a rotating wheel. Beads were washed extensively twice each with Low Salt, High Salt and LiCl Immune Complex Wash Buffer and TE buffer (Upstate). Cross-linking of the eluates was reversed by incubation at $65^{\circ} \mathrm{C}$ overnight, and the precipitated DNA was purified with a PCR purification kit (Qiagen). Onetwenty-fifth of the eluted DNA was used for subsequent PCR; the cycle number varied from 32 to 35 . The primers used to assay for precipitation of genomic DNA were as follows; D (WUS), TCGATAGAAATGCCAAACGAC and GGATTCAA CACAACGTGCAA; P (WUS), TTTCAATCCTTCTCCACCA GA and GGGACCCAAAAAGAAGGTTT; G (WUS), GGCAAA CCAAATTTGCATCTA and TCAGTACCTGAGCTTGCAT GA; D (STM), GGTACAGGTGTCCCATGCTT and AACCAT TTGTATTTGCCACCA; P (STM), TCCGGTTTTCTCCATT TCAA and GAGGGAAGCAGCAGAAGAGA; G (STM), TTC CTTCAACGTGTCGAGTG and TTGTTGTTGTACCAAAG CAAATG; EIF4P, CGCATCCTATCGGATTGTCT and CTCA GATGATGTGCGGAGAA.

\section{Acknowledgments}

We are grateful for critical comments and suggestions by Jennifer Fletcher, Jennifer Pfluger, Scott Poethig, and John Wagner. We also thank Yanhui Su for technical support. This work was supported by National Institutes of Health grant RO1 GM064650-01 to D.W.

\section{References}

Aida, M., Ishida, T., Fukaki, H., Fujisawa, H., and Tasaka, M. 1997. Genes involved in organ separation in Arabidopsis: An analysis of the cup-shaped cotyledon mutant. Plant Cell 9: 841-857.

Aida, M., Ishida, T., and Tasaka, M. 1999. Shoot apical meristem and cotyledon formation during Arabidopsis embryogenesis: Interaction among the CUP-SHAPED COTYLEDON and SHOOT MERISTEMLESS genes. Development
126: $1563-1570$.

Armstrong, J.A., Papoulas, O., Daubresse, G., Sperling, A.S., Lis, J.T., Scott, M.P., and Tamkun, J.W. 2002. The Drosophila BRM complex facilitates global transcription by RNA polymerase II. EMBO J. 21: 5245-5254.

Barton, M.K. and Poethig, R.S. 1993. Formation of the shoot apical meristem in Arabidopsis thaliana: An analysis of development in the wild-type and in the shoot meristemless mutant. Development 119: 823-831.

Baurle, I. and Laux, T. 2003. Apical meristems: The plant's fountain of youth. Bioessays 25: 961-970.

Becker, P.B. and Horz, W. 2002. ATP-dependent nucleosome remodeling. Annu. Rev. Biochem. 71: 247-273.

Bertrand, C., Bergounioux, C., Domenichini, S., Delarue, M., and Zhou, D.X. 2003. Arabidopsis histone acetyltransferase AtGCN5 regulates the floral meristem activity through the WUSCHEL/AGAMOUS pathway. I. Biol. Chem. 278: 28246-28251.

Brand, U., Fletcher, J.C., Hobe, M., Meyerowitz, E.M., and Simon, R. 2000. Dependence of stem cell fate in Arabidopsis on a feedback loop regulated by CLV3 activity. Science 289: 617-619.

Brand, U., Grunewald, M., Hobe, M., and Simon, R. 2002. Regulation of CLV3 expression by two homeobox genes in Arabidopsis. Plant Physiol. 129: 565-575.

Brzeski, J. and Jerzmanowski, A. 2003. Deficient in DNA methylation 1 (DDM1) defines a novel family of chromatin-remodeling factors. J. Biol. Chem. 278: 823-828.

Bultman, S., Gebuhr, T., Yee, D., La Mantia, C., Nicholson, J., Gilliam, A., Randazzo, F., Metzger, D., Chambon, P., Crabtree, G., et al. 2000. A Brg1 null mutation in the mouse reveals functional differences among mammalian SWI/SNF complexes. Mol. Cell 6: 1287-1295.

Byrne, M.E., Barley, R., Curtis, M., Arroyo, J.M., Dunham, M., Hudson, A., and Martienssen, R.A. 2000. Asymmetric leaves1 mediates leaf patterning and stem cell function in Arabidopsis. Nature 408: 967-971.

Carles, C.C. and Fletcher, J.C. 2003. Shoot apical meristem maintenance: The art of a dynamic balance. Trends Plant Sci. 8: 394-401.

Chen, C., Wang, S., and Huang, H. 2000. LEUNIG has multiple functions in gynoecium development in Arabidopsis. Genesis 26: $42-54$.

Clark, S.E. 2001. Cell signalling at the shoot meristem. Nat. Rev. Mol. Cell. Biol. 2: 276-284.

Clark, S.E., Running, M.P., and Meyerowitz, E.M. 1993. CLAVATA1, a regulator of meristem and flower development in Arabidopsis. Development 119: 397-418.

Clark, S.E., Jacobsen, S.E., Levin, J.Z., and Meyerowitz, E.M. 1996. The CLAVATA and SHOOT MERISTEMLESS loci competitively regulate meristem activity in Arabidopsis. Development 122: 1567-1575.

Clark, S.E., Williams, R.W., and Meyerowitz, E.M. 1997. The CLAVATA1 gene encodes a putative receptor kinase that controls shoot and floral meristem size in Arabidopsis. Cell 89: $575-585$.

Damelin, M., Simon, I., Moy, T.I., Wilson, B., Komili, S., Tempst, P., Roth, F.P., Young, R.A., Cairns, B.R., and Silver, P.A. 2002. The genome-wide localization of Rsc9, a component of the RSC chromatin-remodeling complex, changes in response to stress. Mol. Cell 9: 563-573.

Dievart, A., Dalal, M., Tax, F.E., Lacey, A.D., Huttly, A., Li, J., and Clark, S.E. 2003. CLAVATA1 dominant-negative alleles reveal functional overlap between multiple receptor kinases that regulate meristem and organ development. Plant Cell 15: $1198-1211$. 
Emerson, B.M. 2002. Specificity of gene regulation. Cell 109: $267-270$.

Endrizzi, K., Moussian, B., Haecker, A., Levin, J.Z., and Laux, T. 1996. The SHOOT MERISTEMLESS gene is required for maintenance of undifferentiated cells in Arabidopsis shoot and floral meristems and acts at a different regulatory level than the meristem genes WUSCHEL and ZWILLE. Plant I. 10: 967-979.

Eshed, Y., Izhaki, A., Baum, S.F., Floyd, S.K., and Bowman, J.L. 2004. Asymmetric leaf development and blade expansion in Arabidopsis are mediated by KANADI and YABBY activities. Development 131: 2997-3006.

Farrona, S., Hurtado, L., Bowman, J.L., and Reyes, J.C. 2004. The Arabidopsis thaliana SNF2 homolog AtBRM controls shoot development and flowering. Development 131: 4965-4975.

Fletcher, J.C., Brand, U., Running, M.P., Simon, R., and Meyerowitz, E.M. 1999. Signaling of cell fate decisions by CLAVATA3 in Arabidopsis shoot meristems. Science 283: 19111914.

Gallois, J.L., Woodward, C., Reddy, G.V., and Sablowski, R. 2002. Combined SHOOT MERISTEMLESS and WUSCHEL trigger ectopic organogenesis in Arabidopsis. Development 129: 3207-3217.

Gregg, R.G., Willer, G.B., Fadool, J.M., Dowling, J.E., and Link, B.A. 2003. Positional cloning of the young mutation identifies an essential role for the Brahma chromatin remodeling complex in mediating retinal cell differentiation. Proc. Natl. Acad. Sci. 100: 6535-6540.

Groß-Hardt, R., Lenhard, M., and Laux, T. 2002. WUSCHEL signaling functions in interregional communication during Arabidopsis ovule development. Genes \& Dev. 16: 11291138.

Holstege, F.C., Jennings, E.G., Wyrick, J.J., Lee, T.I., Hengartner, C.J., Green, M.R., Golub, T.R., Lander, E.S., and Young, R.A. 1998. Dissecting the regulatory circuitry of a eukaryotic genome. Cell 95: 717-728.

Jeong, S., Trotochaud, A.E., and Clark, S.E. 1999. The Arabidopsis CLAVATA2 gene encodes a receptor-like protein required for the stability of the CLAVATA1 receptor-like kinase. Plant Cell 11: 1925-1934.

Kadam, S. and Emerson, B.M. 2003. Transcriptional specificity of human SWI/SNF BRG1 and BRM chromatin remodeling complexes. Mol. Cell 11: 377-389.

Kaya, H., Shibahara, K.I., Taoka, K.I., Iwabuchi, M., Stillman, B., and Araki, T. 2001. FASCIATA genes for chromatin assembly factor-1 in Arabidopsis maintain the cellular organization of apical meristems. Cell 104: 131-142.

Kennison, J.A. and Tamkun, J.W. 1988. Dosage-dependent modifiers of Polycomb and Antennapedia mutations in Drosophila. Proc. Natl. Acad. Sci. 85: 8136-8140.

Laux, T., Mayer, K.F., Berger, J., and Jurgens, G. 1996. The WUSCHEL gene is required for shoot and floral meristem integrity in Arabidopsis. Development 122: 87-96.

Lenhard, M. and Laux, T. 2003. Stem cell homeostasis in the Arabidopsis shoot meristem is regulated by intercellular movement of CLAVATA3 and its sequestration by CLAVATA1. Development 130: 3163-3173.

Lenhard, M., Jurgens, G., and Laux, T. 2002. The WUSCHEL and SHOOTMERISTEMLESS genes fulfill complementary roles in Arabidopsis shoot meristem regulation. Development 129: 3195-3206.

Liu, C.M. and Meinke, D.W. 1998. The titan mutants of Arabidopsis are disrupted in mitosis and cell cycle control during seed development. Plant J. 16: 21-31.

Long, J.A., Moan, E.I., Medford, J.I., and Barton, M.K. 1996. A member of the KNOTTED class of homeodomain proteins encoded by the STM gene of Arabidopsis. Nature 379: 66-69.

Lorch, Y., Zhang, M., and Kornberg, R.D. 1999. Histone octamer transfer by a chromatin-remodeling complex. Cell 96: 389392.

Lusser, A. and Kadonaga, J.T. 2003. Chromatin remodeling by ATP-dependent molecular machines. Bioessays 25: 1192-1200.

Martens, J.A. and Winston, F. 2003. Recent advances in understanding chromatin remodeling by Swi/Snf complexes. Curr. Opin. Genet. Dev. 13: 136-142.

Mayer, K.F., Schoof, H., Haecker, A., Lenhard, M., Jurgens, G., and Laux, T. 1998. Role of WUSCHEL in regulating stem cell fate in the Arabidopsis shoot meristem. Cell 95: 805-815.

Moehs, C.P., McElwain, E.F., and Spiker, S. 1988. Chromosomal proteins of Arabidopsis thaliana. Plant Mol. Biol. 11: 507515.

Narlikar, G.J., Fan, H.Y., and Kingston, R.E. 2002. Cooperation between complexes that regulate chromatin structure and transcription. Cell 108: 475-487.

Neff, M.M., Neff, J.D., Chory, J., and Pepper, A.E. 1998. dCAPS, a simple technique for the genetic analysis of single nucleotide polymorphisms: Experimental applications in Arabidopsis thaliana genetics. Plant T. 14: 387-392.

Ng, H.H., Robert, F., Young, R.A., and Struhl, K. 2002. Genomewide location and regulated recruitment of the RSC nucleosome-remodeling complex. Genes \& Dev. 16: 806-819.

Orlando, V. 2000. Mapping chromosomal proteins in vivo by formaldehyde-crosslinked-chromatin immunoprecipitation. Trends Biochem. Sci. 25: 99-104.

Peterson, C.L. and Workman, J.L. 2000. Promoter targeting and chromatin remodeling by the SWI/SNF complex. Curr. Opin. Genet. Dev. 10: 187-192.

Reyes, J.C., Barra, J., Muchardt, C., Camus, A., Babinet, C., and Yaniv, M. 1998. Altered control of cellular proliferation in the absence of mammalian brahma (SNF2 $\alpha$ ). EMBO $J$. 17: 6979-6991.

Rojo, E., Sharma, V.K., Kovaleva, V., Raikhel, N.V., and Fletcher, J.C. 2002. CLV3 is localized to the extracellular space, where it activates the Arabidopsis CLAVATA stem cell signaling pathway. Plant Cell 14: 969-977.

Sawa, H., Kouike, H., and Okano, H. 2000. Components of the SWI/SNF complex are required for asymmetric cell division in C. elegans. Mol. Cell 6: 617-624.

Schoof, H., Lenhard, M., Haecker, A., Mayer, K.F., Jurgens, G., and Laux, T. 2000. The stem cell population of Arabidopsis shoot meristems is maintained by a regulatory loop between the CLAVATA and WUSCHEL genes. Cell 100: 635-644.

Sengupta, S.M., VanKanegan, M., Persinger, J., Logie, C., Cairns, B.R., Peterson, C.L., and Bartholomew, B. 2001. The interactions of yeast SWI/SNF and RSC with the nucleosome before and after chromatin remodeling. I. Biol. Chem. 276: 12636-12644.

Sieburth, L.E. and Meyerowitz, E.M. 1997. Molecular dissection of the AGAMOUS control region shows that cis elements for spatial regulation are located intragenically. Plant Cell 9: 355-365.

Sudarsanam, P., Iyer, V.R., Brown, P.O., and Winston, F. 2000. Whole-genome expression analysis of snf/swi mutants of Saccharomyces cerevisiae. Proc. Nat1. Acad. Sci. 97: 3364 3369.

Takada, S., Hibara, K., Ishida, T., and Tasaka, M. 2001. The CUP-SHAPED COTYLEDON1 gene of Arabidopsis regulates shoot apical meristem formation. Development 128: $1127-1135$

Takeda, S., Tadele, Z., Hofmann, I., Probst, A.V., Angelis, K.J., Kaya, H., Araki, T., Mengiste, T., Scheid, O.M., Shibahara, 
K., et al. 2004. BRU1, a novel link between responses to DNA damage and epigenetic gene silencing in Arabidopsis. Genes \& Dev. 18: 782-793.

Trotochaud, A.E., Hao, T., Wu, G., Yang, Z., and Clark, S.E. 1999. The CLAVATAl receptor-like kinase requires CLAVATA3 for its assembly into a signaling complex that includes KAPP and a Rho-related protein. Plant Cell 11: 393406.

Tsiantis, M. and Hay, A. 2003. Comparative plant development: The time of the leaf? Nat. Rev. Genet. 4: 169-180.

Tsukiyama, T. 2002. The in vivo functions of ATP-dependent chromatin-remodelling factors. Nat. Rev. Mol. Cell. Biol. 3: 422-429.

Verbsky, M.L. and Richards, E.J. 2001. Chromatin remodeling in plants. Curr. Opin. Plant Biol. 4: 494-500.

Vroemen, C.W., Mordhorst, A.P., Albrecht, C., Kwaaitaal, M.A., and de Vries, S.C. 2003. The CUP-SHAPED COTYLEDON3 gene is required for boundary and shoot meristem formation in Arabidopsis. Plant Cell 15: 1563-1577.

Wagner, D. and Meyerowitz, E.M. 2002. SPLAYED, a novel SWI/SNF ATPase homolog, controls reproductive development in Arabidopsis. Curr. Biol. 12: 85-94.

William, D.A., Su, Y., Smith, M.R., Lu, M., Baldwin, D.A., and Wagner, D. 2004. Genomic identification of direct target genes of LEAFY. Proc. Natl. Acad. Sci. 101: 1775-1780.- 


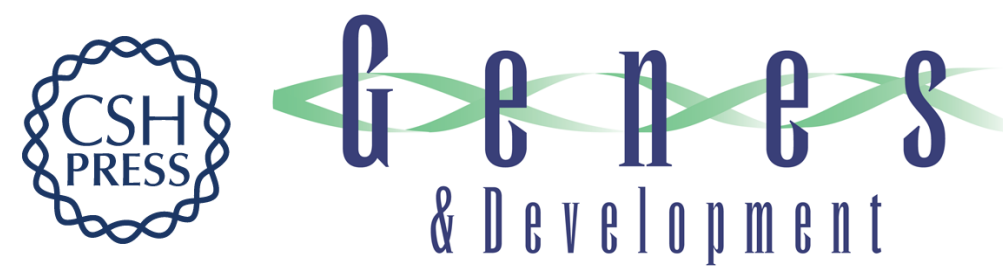

\section{WUSCHEL is a primary target for transcriptional regulation by SPLAYED in dynamic control of stem cell fate in Arabidopsis}

Chang Seob Kwon, Changbin Chen and Doris Wagner

Genes Dev. 2005, 19:

Access the most recent version at doi:10.1101/gad.1276305

Supplemental
Material http://genesdev.cshlp.org/content/suppl/2005/04/01/19.8.992.DC1

References This article cites 64 articles, 33 of which can be accessed free at:

http://genesdev.cshlp.org/content/19/8/992.full.html\#ref-list-1

License

Email Alerting Receive free email alerts when new articles cite this article - sign up in the box at the top

Service right corner of the article or click here.

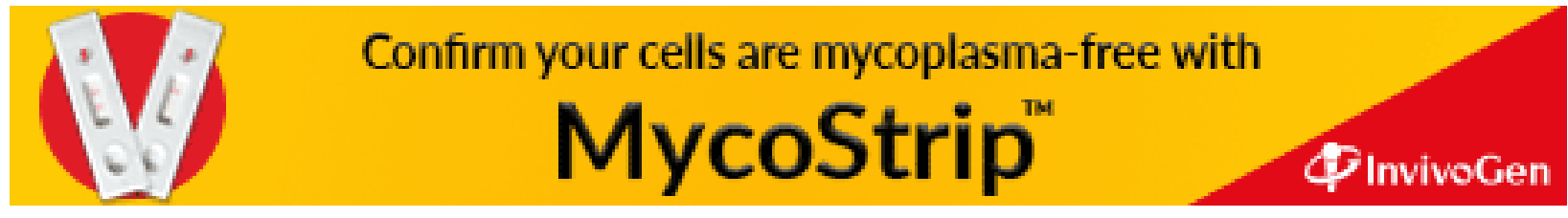

